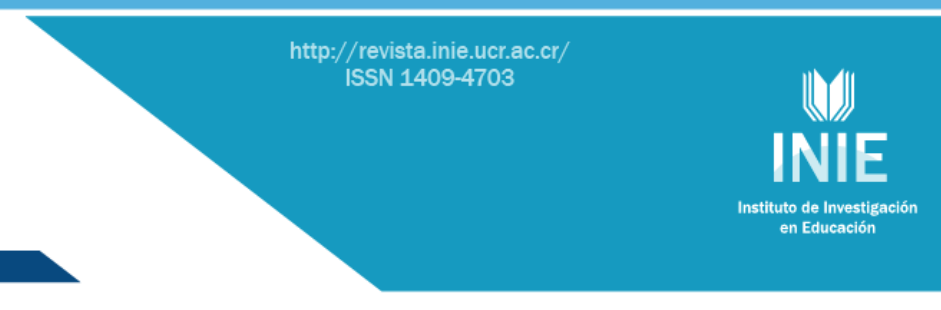

\title{
VIOLENCIA ESCOLAR, UN PROBLEMA QUE AUMENTA EN LA
} ESCUELA PRIMARIA COSTARRICENSE

SCHOOL VIOLENCE, AN INCREASING PROBLEM IN COSTARRICAN ELEMENTARY SCHOOLS

\author{
Volumen 13, Número 2 \\ Mayo - Agosto \\ pp. $1-20$
}

Este número se publicó el 30 de mayo de 2013

Hannia Cabezas Pizarro

Melania Monge Rodríguez

Revista indizada en REDALYC, $\underline{\text { SCIELO }}$

Revista distribuida en las bases de datos:

CATÁLOGO DE LATINDEX, IRESIE, CLASE, DIALNET, DOAJ, E-REVIST@S, SHERPA/ROMEO, QUALIS, MIAR

Revista registrada en los directorios:

ULRICH'S, REDIE, RINACE, OEI, MAESTROTECA, PREAL, $\underline{\text { CLASCO }}$ 


\title{
VIOLENCIA ESCOLAR, UN PROBLEMA QUE AUMENTA EN LA ESCUELA PRIMARIA COSTARRICENSE SCHOOL VIOLENCE, AN INCREASING PROBLEM IN COSTARRICAN ELEMENTARY SCHOOLS
}

\author{
Hannia Cabezas Pizarro ${ }^{1}$ \\ Melania Monge Rodríguet
}

\begin{abstract}
Resumen: En este artículo se compararon los datos de investigaciones previas, sobre la presencia del matonismo escolar en muestras costarricenses, con los datos obtenidos en un estudio reciente, cuyo fin fue el de determinar si este fenómeno había aumentado en el país. Para ello se seleccionaron de forma intencional, 34 grupos de niños y niñas cuyas edades se encontraban entre los 8 y 15 años quienes cursaban estudios de tercero a sexto grado, en diferentes centros educativos, para un total de 1155 estudiantes. Una vez contabilizadas las preguntas y transformadas a porcentajes de respuestas, se compararon con los porcentajes obtenidos en estudios realizados en Costa Rica por Cabezas $(2007,2010)$ en grupos similares, hallándose diferencias significativas que hacen presumir que ésta es una realidad, que va en aumento en el país.
\end{abstract}

Palabras clave: MALTRATO ENTRE IGUALES, INCREMENTO DE VIOLENCIA, BULLYING, SISTEMA EDUCATIVO, COSTA RICA

\begin{abstract}
This article compares previous results with those from a recent research on bullying among school children in Costa Rica to determine if this undesirable behavior had increased in the country. We selected, intentionally, 34 groups of elementary students, third through sixth grade, ages 8 through 15 years old, from different schools. The total of students participating was 1155. They were given the same survey and once the tally was converted to percentages of responses, they were compared with results obtained from previous studies done in different regions of Costa Rica by Cabezas (2007, 2010), under similar circumstances. This new results show significant differences that make us presume that bullying is a reality and is increasing in Costa Rican schools
\end{abstract}

Key words: BULLYING, AGGRESSION AMONG EQUALS, ABUSE OF POWER, EDUCATIONAL SYSTEM, COSTA RICA

\footnotetext{
${ }^{1}$ Docente de la Escuela de Orientación y Educación Especial, Facultad de Educación, Universidad de Costa Rica. Maestría en Rehabilitación Integral; Licenciada en Administración Educativa; Bachiller en Retardo Mental de la Escuela de Orientación y Educación Especial, todos los títulos de la Universidad de Costa Rica. Dirección electrónica: hanniac@gmail.com

${ }^{2}$ Docente de la Escuela de Orientación y Educación Especial, Facultad de Educación, Universidad de Costa Rica. Doctoranda en Neuropsicología Clínica de la Universidad de Salamanca, Maestría en Problemas de Aprendizaje de la Universidad Estatal de lowa, Bachiller en Educación Primaria de la Universidad de Costa Rica. Dirección electrónica:melania.monge@ucr.ac.cr
}

Artículo recibido: 18 de octubre, 2012

Aprobado: 20 de mayo, 2013 


\section{Introducción}

Las investigaciones realizadas en países como los Estados Unidos y Noruega (Nansel, Overpeck, Ruean, Simons, y Scheidt, 2001) indican que en cada uno de ellos, la presencia del acoso escolar tiene características propias que responden a las diferencias históricas y culturales de cada país. Como lo plantea Monclús (2005) "la violencia en la escuela es, en efecto, reflejo de la sociedad" (p. 4); sin embargo, es necesario hacer distinción entre la violencia en general y el acoso escolar, porque también existen criterios claros que determinan las diversas formas de violencia.

En el perfil de las víctimas y los victimarios, así como en los porcentajes de maltrato escolar hallados en las muestras costarricenses, no se encontraron discrepancias significativas, ya que estos estudios realizados en los años 2007 y 2010 reflejan, también, las características propias del bullying, tanto en la forma de maltrato como en la prevalencia del fenómeno.

En promedio, la presencia de conductas agresivas en el salón de clases, en forma general, según Olweus (1993), se estima en un 15\%, sin embargo este porcentaje varía entre los estudios realizados por Craig y otros (2009) en países del Norte de Europa y países Bálticos donde se encuentran discrepancias que van desde $8.6 \%$ al $45.2 \%$ en el caso de los varones y de un $4.8 \%$ a $35.8 \%$ en el caso de las mujeres.

En Costa Rica de acuerdo con Cabezas $(2007,2010,2013)$ el $10.2 \%$ de los y las estudiantes encuestadas manifestaron agredir a sus iguales, mientras que un $12 \%$ dijo haber sido víctimas, tornándose en una situación preocupante donde las figuras de autoridad en los centros educativos deben prestar especial atención y actuar de manera inmediata, explorando estrategias que permitan brindar un abordaje integral desde el punto de vista educativo y el de salud.

El bullying en el aula crece, en primer lugar, porque el docente, sin proponérselo, puede estimular la presencia de conductas agresivas entre sus estudiantes al no reconocer que el maltrato entre iguales es real, y los actos llevados a cabo por las alumnas y los alumnos pueden estar siendo reforzados por la tolerancia, la pasividad, la ausencia en la toma de decisiones y el no establecimiento de reglas claras dentro del salón de clases.

En el trabajo realizado por Cabezas, (2007) se encontró que en un 59.5\% de las veces en las que el estudiantado fue sometido, con la intención de causarle daño, quienes agredieron no recibieron ningún castigo por parte de las personas con investidura de 
autoridad, lo que refuerza el hecho al no darse una corrección inmediata sobre las conductas agresivas que manifestaron los y las estudiantes en el aula.

Otro factor que incide en el aumento del bullying es el social, donde la exclusión, la discriminación, la inseguridad, la iniquidad económica, la insatisfacción personal, así como los niveles de pobreza, además del entorno, pueden convertirse en desencadenantes de comportamientos aprendidos en el hogar o en la comunidad, que se trasladan al salón de clases.

\section{Marco Referencial}

\section{Maltrato escolar y población costarricense}

Las acciones violentas se manifiestan de diversas formas y en muchos escenarios del quehacer costarricense. En las carreteras, por ejemplo, es posible observar la conducción temeraria de algunas personas en distintos tipos de vehículos. De igual forma, en los sitios de trabajo se experimentan diversas maneras de abuso de poder, tales como, acoso o discriminación; sin dejar de lado los asaltos, la invasión a la propiedad en las distintas comunidades y la agresión física y emocional presente en muchos hogares.

En ese sentido, la violencia no solo se manifiesta con los golpes, sino bajo condiciones más dañinas que involucran palabras humillantes, el temor, la indiferencia, el abuso de poder, la hostilidad, la exclusión u otros modos menos evidentes que llevan al sometimiento.

De acuerdo con Menesini y Nocentini (2009), un 20\% de la población estudiantil está involucrada en el acoso escolar; esta forma de violencia no solo se manifiesta a través de los golpes, también se presenta como acoso verbal y en particular el acoso homofóbico, hacia los compañeros y compañeras. "Estos comportamientos, de tipo verbal, físico sexual e indirecto, tienen una fuerte interconexión con el acoso escolar, puesto que a menudo, en la primera fase de la adolescencia, las primeras experiencias de citas tienen lugar al interior del grupo y la calidad de la relación con la pareja sufre fuertemente los efectos de la calidad de las relaciones de amistad y sociales en el grupo referencia" (Menesine y Nocentini, 2009, p.10).

Investigaciones realizadas por Currie, Gabhainn, y Godeau (2008), con niños, niñas y adolescentes europeos, indican que la proporción de quienes son víctimas de sus compañeros es de un 20.6\%, y específicamente en España, el porcentaje alcanza el 23.7\%, datos que sobrepasan la media dada por Olweus en (1993), pionero en el tema, porque 
reflejan que este fenómeno ha ido en aumento con el paso del tiempo, no solo en nuestro país, sino también en países europeos como España. Algunos indicadores que nos permiten hacer comparaciones son los aportados por Cid, Díaz, Pérez, Torruella y Valderrama (2008), quienes encontraron que, en Chile, el $45 \%$ de los estudiantes, con edades entre 10 y 13 años, manifestaron haber sido agredidos por sus iguales.

Por su parte, Ng. W.Y. y Tsang (2008), también hacen referencia a los porcentajes que se mantienen a través de diversas investigaciones, como es el caso del "17\% en Australia; $19 \%$ en Inglaterra; $15 \%$ en Japón; 14\% en Noruega y $16 \%$ en los Estados Unidos" (p. 4). Estos estudios fueron realizados con víctimas de agresión escolar, quienes manifestaron haber experimentado el maltrato una o dos veces en los últimos cuatro meses, cuando se llevaba a cabo el estudio.

En trabajos más recientes, Nansel, Overpeck, Pilla, Ruan, Simons-Morton y Scheidt (2001) reportaron que alrededor de un $30 \%$ de los estudiantes habían estado involucrados en situaciones de maltrato escolar, ya sea como víctimas o como victimarios.

Los aportes costarricenses con muestras de niños y adolescentes evidencian indicadores que sobrepasan la media europea. Los datos obtenidos en los estudios nacionales varían desde el 19.1\% (Cabezas y Monge 2007), 14.3\% (Cabezas, 2010) hasta 20.35\% (Cabezas, 2013).

Las investigaciones nacionales describen, a la vez, los diversos calificativos empleados por las personas que victimizan a sus iguales, entre los que sobresalen: "tontas", "débiles," "fáciles de manipular", que coinciden con la aportada inicialmente por Olweus (1993), quien anotó, además, como una característica del fenómeno del bullying, que existe un desequilibrio de poder, condición necesaria para calificar al acoso entre iguales, y que también se encuentra presente en los estudios costarricenses. Cuando los victimarios identifican a sus compañeros y compañeras como personas "tontas, débiles o fáciles de manipular", estos calificativos apuntan a que las víctimas oponen poca resistencia ante sus agresores, que su ánimo cede fácilmente por la insistencia de otros, o se ven como personas frágiles e indefensas convirtiéndose los niños y niñas en presa fácil para los victimarios.

Si un niño o niña se maltrata o avergüenza delante de sus iguales, los efectos negativos se hacen patentes a corto o largo plazo, porque la violencia conlleva un daño moral y psicológico, que deja secuelas que perduran por muchos años. Al respecto, Clarke y 
Kiselica (citados en Aluede, Adeleke, Omoike, Afen-Akpaida, (2008) mencionan que los sentimientos de soledad y la baja autoestima parecen mantenerse hasta la edad adulta.

De hecho, la transición de la niñez hacia la adolescencia es una época esencial en la vida de todo ser humano, es una etapa de autoafirmación que prepara a la juventud para la vida adulta. Si la seguridad y autoestima se ven debilitadas en el ser humano, también se debilita la capacidad para tomar decisiones en relación con la escuela y los amigos, y podrían repetir, a través de su vida, un rol que les ha sido impuesto (Olweus, 1993; Kumpulainen y Räsänen, 2000). De acuerdo con los mismos autores, en la adultez tanto el agresor como la víctima muestran dificultades para establecer relaciones interpersonales sanas y positivas, ya que "las condiciones en las que una persona atraviesa la infancia y la adolescencia determinarán características, capacidades y habilidades personales que tendrán influencia en sus relaciones vinculares, sus posibilidades de inserción social y su rendimiento laboral, entre otros aspectos de la vida adulta" (Pincever, 2008, p. 102).

Asimismo, las interacciones sociales que las niñas y niños establecen con sus pares, y personas adultas significativas en el ámbito escolar, van a influir de manera directa en la percepción que desarrollen de sí mismos, así como en la calidad de las relaciones interpersonales, la motivación hacia el aprendizaje y el rendimiento académico. Si la persona experimenta vivencias negativas, como la violencia escolar, generalmente, las repercusiones en su desarrollo podrían ser nefastas.

En esta dirección, los agresores o victimarios suelen presentar desajustes socioemocionales de tipo crónico. Algunas de las características que pueden darse son: la falta de empatía, la ausencia de arrepentimiento, la mala regulación emocional o la manifestación de conductas agresivas e impulsivas, conductas delictivas y drogadicción, afectando a los niños y a las niñas, en su entorno inmediato y en su comportamiento en el aula (O’Brennan, Bradshaw y Sawyer, 2009).

En ese sentido, Sourander, Jensen, Rönning, Niemelä, Helenius, Sillanmäki, Kumpulainen, Piha, Tamminen, Moilanen, y Almqvist (2007b), encontraron que existe una relación entre edad y frecuencia en la manifestación de las conductas de maltrato escolar, lo que significa que entre más temprano se desarrollen dichas conductas y la reiteración con que se presenten, mayor es el riesgo que tiene la persona de manifestar desórdenes de personalidad y conductas delictivas en la edad adulta. 
Por lo anterior, este estudio tiene vital importancia, porque permite comparar los índices de acoso escolar en diferentes muestras costarricenses, en las que se evidencia que el maltrato entre iguales, lejos de ser un fenómeno que desaparece con el tiempo, aumenta de manera significativa en escolares costarricenses.

\section{Objetivo}

El objetivo general de esta investigación fue determinar la situación del acoso escolar en Costa Rica, partiendo de estudios previos realizados en el país, y del análisis comparativo con datos actuales.

\section{Metodología}

Este es un estudio cuantitativo tipo encuesta, donde la información se recolectó, aplicando un instrumento, que también fue administrado en estudios previos (Cabezas y Monge, 2007; Cabezas, 2011; Paredes, Lega, y Cabezas, 2011), a un nuevo grupo de estudiantes matriculados en los niveles de tercero a sexto grado en varios centros educativos costarricenses. Al realizar el estudio, los alumnos y las alumnas correspondieron a distintas escuelas localizadas en Escazú, San José, Alajuela, Desamparados, Moravia, Tirrases de Curridabat, San Rafael de Montes de Oca, Cartago y Guadalupe, en la provincia de San José, Costa Rica. Cuando se administraron los instrumentos se les explicó qué era lo que se pretendía con el estudio; además, se les motivó para que brindaran las respuestas sin temor, indicándoseles que debían responder el cuestionario en forma individual y anónima, lo que facilitó que las respuestas fueran sinceras.

El cuestionario que se administró consta de preguntas abiertas y cerradas, donde se incluyen los criterios establecidos a nivel mundial para determinar la presencia del bullying en el aula. La construcción teórica del documento fue revisada por expertos, quienes le dieron validez tanto de construcción como de contenido. El instrumento se ha comportado de manera consistente en las diversas muestras seleccionadas, reflejando su validez.

Para efectos de esta investigación, el cuestionario se administró a 34 grupos de niños, niñas y adolescentes con edades entre los 8 y 15 años, que cursaban estudios de tercero a sexto grado, como se dijo, en diferentes centros educativos del país, para un total de 1155 instrumentos aplicados. 
Una vez contabilizadas las preguntas y realizadas las conversiones de los datos arrojados por las respuestas, en porcentajes, los resultados se compararon con estudios previos realizados en Costa Rica por Cabezas, durante el 2007 y el 2010, con grupos similares. Para el año 2007, la muestra seleccionada fue de 371 estudiantes; y en el 2010 de 916. Los resultados obtenidos en estas investigaciones se anotaron, compararon y analizaron posteriormente con los datos obtenidos en el año 2012 (Ver Tabla 1).

\section{Análisis de la Información}

Para una mejor comprensión de los resultados en las frecuencias obtenidas, se convirtieron a porcentajes de respuesta. Se elaboraron tablas y gráficos, donde se anotaron los indicadores que se pretendían medir y comparar, acompañados de los porcentajes de respuestas obtenidos en cada uno de los estudios realizados en los años: 2007, 2010 y el más reciente estudio correspondiente a 2012. (Ver Tabla y Gráfico 1).

Tabla 1

Porcentajes obtenidos en estudiantes de secundaria y primaria que mostraron conductas amenazantes en los años 2007, 2010 y 2012

\begin{tabular}{|c|c|c|}
\hline $\begin{array}{c}\text { Entre 13 y 16 años } \\
(2007)\end{array}$ & Entre 9 y 14 años & Entre 8 y 14 años \\
$(2010)$ & $2012)$ \\
\hline $17.1 \%$ & $14.3 \%$ & $20.35 \%$ \\
\hline
\end{tabular}

\section{Gráfico 1}

Porcentajes obtenidos en estudiantes de secundaria y primaria que mostraron conductas amenazantes en los años 2007, 2010 y 2012

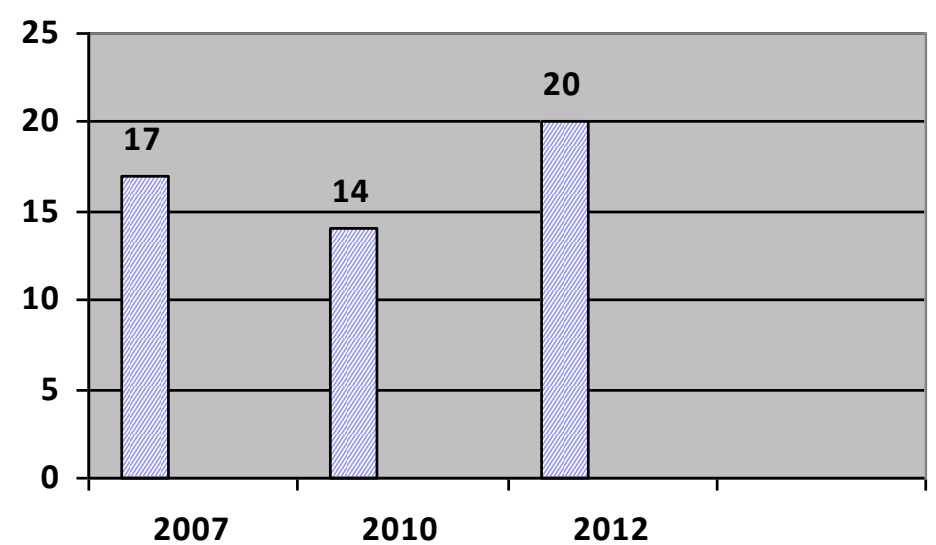




\section{Comentario}

Como puede observarse, tanto en la Tabla 1 como en el Gráfico 1, el acoso escolar va en aumento. Esta realidad afecta a los estudiantes de muchos países del mundo, y Costa Rica no está exenta.

El aumento del bullying podría relacionarse con la problemática social del país y las manifestaciones de conductas agresivas del entorno donde se desarrollan los niños y las niñas.

En la Tabla 1 también puede apreciarse la significativa diferencia de edad entre el estudiantado. Es importante resaltar que estas diferencias de edad se encuentran dentro de un mismo salón de clases, debido a que algunos niños y niñas han venido repitiendo el año escolar en varias ocasiones, situación que los va convirtiendo en líderes del grupo.

Al ser mayores que el resto de sus iguales, físicamente más grandes, y al acumular experiencias de años anteriores, se establecen relaciones desiguales con los más pequeños, de modo que la diferencia de edad se convierte en un elemento más que debe controlar el docente y en un factor desequilibrante que perturba el ambiente del aula, ya que genera conflictos.

Además, podrían sumarse al aumento del bullying en el aula, otros factores como: los pocos límites que tiene la población infantil y el pobre abordaje que se hace del tema en la escuela, por lo que podría deducirse que la violencia también se extiende a diversos ambientes de convivencia diaria, como el laboral, el recreativo y el familiar.

Otro lugar a tomar en consideración es la escuela, porque es un sitio de convivencia donde hay diversidad de pensamiento y donde se suman las diferencias en la formación familiar, las características sociales propias de cada educando, además de la gran cantidad de estudiantes matriculados por sección. Todos estos factores ayudan a que el recinto escolar se convierta en un lugar propicio para que afloren las controversias entre el alumnado y se manifieste una lucha de intereses, donde prevalece la voluntad del más fuerte, quien abusa mediante la fuerza de sus compañeros y compañeras, les intimida con un lenguaje amenazante e insulta; además, tiende a mostrar un comportamiento agresivo con el que somete a las víctimas atemorizándolas y obligándolas a hacer cosas que no desean.

Los datos obtenidos a través de los estudios costarricenses pueden compararse también con investigaciones realizadas en países desarrollados como los Estados Unidos, 
de las que se desprende que un $24 \%$ de la población encuestada ha victimizado de una u otra forma a sus compañeros o compañeras (Nansel et al., 2001).

Si se toma en consideración que quienes intimidan a los iguales no han desarrollado empatía hacia ellos, por lo que no pueden imaginar la angustia que experimentan, podría interpretarse que la ausencia de esta conciencia, aunada al poco abordaje que se da en el aula de esta problemática, conlleva a una mayor probabilidad de que aumente el acoso escolar con consecuencias cada vez más nocivas.

Es interesante resaltar que los datos que se representan en la Tabla 2 y Gráfico 2 difieren, en gran medida, de los brindados por las víctimas para las mismas investigaciones.

Tabla 2

Comparación de los datos de estudiantes de secundaria y primaria que manifiestan haber sido víctimas o victimarios de sus compañeros, en los años 2007, 2010, 2012

\begin{tabular}{|l|c|c|}
\hline $\begin{array}{c}\text { Entre 13 y 16 años } \\
(2007)\end{array}$ & $\begin{array}{c}\text { Entre } 9 \text { y } 14 \text { años } \\
(2010)\end{array}$ & $\begin{array}{c}\text { Entre } 8 \text { y } 14 \text { años } \\
(2012)\end{array}$ \\
\hline Víctimas $32.6 \%$ & $29 \%$ & $41 \%$ \\
\hline Victimario $\quad 19.1 \%$ & $14.3 \%$ & $20.35 \%$ \\
\hline
\end{tabular}

\section{Gráfico 2}

Comparación de los datos de estudiantes de secundaria y primaria que manifiestan haber sido víctimas o victimarios de sus compañeros, en los años 2007, 2010, 2012

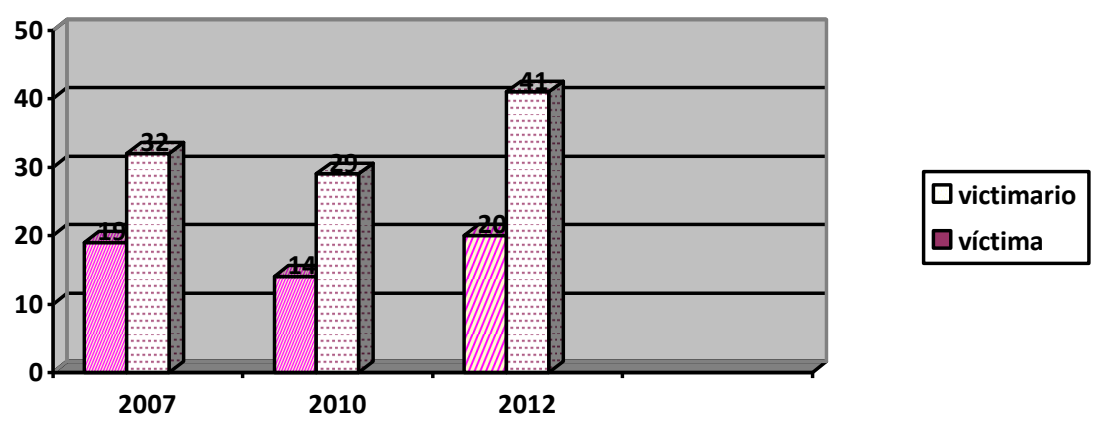

\section{Comentario}

El estudio realizado por Cabezas, durante el 2007, muestra que un $17.1 \%$ de las personas participantes agredieron a sus iguales, mientras que un $32.6 \%$ de las personas de ese mismo grupo indicó haber sido víctima. En el segundo estudio, realizado en el año 2010, un $14.3 \%$ de la población estudiada anotó haber agredido a sus compañeros o compañeras, mientras que un $29 \%$ de esa misma población señaló haber sido víctima de 
ellos. En la última investigación, solo un 20.35\% dijo haber maltratado a sus iguales, mientras que para ese mismo grupo etario un $41 \%$ manifestó haber sido victimizado por sus iguales.

El aumento de un $29 \%$ a $41 \%$ en estudios similares es preocupante, ya que el salón de clases puede convertirse en un lugar atemorizante, que puede afectar el desarrollo social, emocional y moral del estudiantado. Al comparar los datos de referencia con los obtenidos en el 2012, se desprende que la violencia física y los apodos son las formas utilizadas con mayor frecuencia por niños y niñas para agredir a sus iguales.

Los niños y las niñas también aprenden de la interacción diaria. Con esta convivencia se fortalecen las actitudes, se manejan emociones y se desarrolla el autocontrol. Los acontecimientos en el aula forman parte de la cotidianeidad del estudiantado que influye en la personalidad de cada uno.

La evidencia muestra que los niños y niñas que han sido víctimas de sus iguales o que han agredido a sus contrapartes podrían manifestar consecuencias emocionales que marcan sus vidas a corto, mediano y largo plazo. Los efectos de la violencia escolar en la salud mental de la población estudiantil tiene un impacto negativo, sobre todo, en la víctima, ya que transgrede el derecho a la dignidad y seguridad.

El sometimiento físico y psicológico, de forma reiterada, crea un patrón de miedo, culpabilidad e inseguridad en la víctima. Los índices de agresión que se manifiestan en los tres estudios costarricenses muestran que la escuela podría convertirse en un lugar inseguro, donde probablemente no se están tomando las acciones adecuadas para mantener la integridad de los alumnos y alumnas que asisten a ellas.

Las formas de agresión entre iguales más frecuentes se caracterizan por los golpes, las amenazas y los insultos. Estos datos pueden observarse, a continuación, en la Tabla 3 y en el Gráfico 3.

Tabla 3

Formas más frecuentes de agresión en estudiantes por rango de edad

\begin{tabular}{|l|c|c|}
\hline $\begin{array}{c}\text { Entre 13 y 16 años } \\
(2007)\end{array}$ & $\begin{array}{c}\text { Entre 9 y 14 años } \\
(2010)\end{array}$ & $\begin{array}{c}\text { Entre 8 y 14 años } \\
(2012)\end{array}$ \\
\hline Golpes $\quad 32 \%$ & $55 \%$ & $21 \%$ \\
\hline Amenazas $\quad 39 \%$ & $31 \%$ & $23 \%$ \\
\hline Humillar insultar $26 \%$ & $13 \%$ & $23 \%$ \\
\hline Arma blanca o fuego $1 \%$ & & \\
\hline
\end{tabular}




\section{Gráfico 3}

Formas más frecuentes de agresión en estudiantes por rango de edad

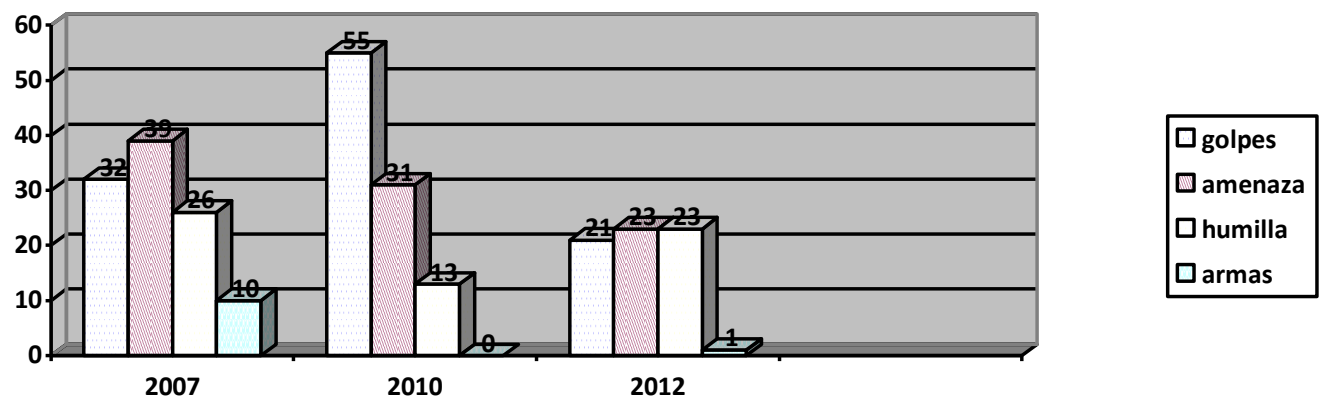

\section{Comentario}

Se han encontrado tres formas de maltrato entre iguales, que presentan mayor frecuencia. En primer lugar, se ubican aquellas que conllevan al maltrato físico, dentro de las que pueden citarse: los puñetazos, las patadas y jalar el cabello. En segundo lugar, están las burlas, destacándose los insultos, los apodos, los gritos y, por último, las amenazas con un factor agregado que es el uso de arma de fuego y arma blanca. Todos estos factores llevan a las víctimas al aislamiento y al retraimiento social.

Como puede apreciarse, la violencia se presenta con mayor agresividad, ya que el uso de armas, como pistolas, cuchillos y navajas en el recinto escolar agrava más la situación. El dominio de unos sobre otros, a través de la fuerza, pareciera ser la forma preferida de un sector de la población estudiantil para intimidar al resto de sus pares. Si bien es cierto son los menos quienes lo han hecho, podría aumentar el porcentaje si el grupo social lo imita, convirtiéndose en un problema mayor para el ambiente de aula. A la vez, el temor que genera en muchos estudiantes el maltrato en la escuela, les puede llevar a creer, erróneamente, que el uso de armas de fuego les protegerá ante la intimidación de otras personas, o que les permitirá controlar al resto del grupo de manera eficaz.

En diversas ocasiones, los diarios y otros medios de comunicación nacionales han informado del enfrentamiento entre estudiantes en los que ha mediado el uso de navajas o pistolas, por lo que el dato encontrado en esta investigación no es sorpresivo para la administración escolar u otras figuras de autoridad. Este hecho ha llevado a implementar medidas de seguridad en diversos centros educativos, como la revisión de mochilas antes de que los alumnos y alumnas ingresen al recinto escolar. 
Entonces, podríamos decir que la violencia se ha incrementado, y que se muestra como reflejo de la sociedad en la que vivimos.

Son muchas y variadas las razones que llevan a los niños y niñas a agredir a sus iguales, las que van desde la diversión, el querer defenderse, por simple gusto o porque les cae mal la víctima escogida, tal y como se puede apreciar en la Tabla 4.

Tabla 4

Razones del estudiantado que impulsan a la agresión de pares

\begin{tabular}{|l|l|l|}
\hline $\begin{array}{l}\text { Adolescentes entre 13 y 16 años } \\
(2007)\end{array}$ & $\begin{array}{l}\text { Niños entre 9 y 14 años } \\
(2010)\end{array}$ & $\begin{array}{l}\text { Niños entre 9 y 14 años } \\
(2012)\end{array}$ \\
\hline Por vacilar & Por defenderme & Le cae mal \\
Me molesta & Por gusto & Me molestaba \\
Me cae mal & Por diversión & Es pequeña \\
Cólera & Es más débil & Porque sí \\
Por pasivo & Me cae mal & Me quitó un lápiz \\
Por miedo & Quería algo suyo & \\
Me insultó & Por venganza & \\
Para que no me amenazara & & \\
Tenía algo mío & & \\
\hline
\end{tabular}

\section{Comentario}

El sentimiento de malestar, por parte de quienes agreden a sus víctimas, se detectó en los tres estudios mencionados. Pareciera que la cultura de intimidación está establecida hacia quienes destacan o se diferencian del resto del grupo. Dentro de las principales características halladas se encontraron: la pasividad, la debilidad, el miedo, la estatura o buenas calificaciones; sin embargo, estas condiciones que aparecen en los estudios caracterizando a las víctimas, no eximen a quienes no las poseen, ya que cualquier estudiante puede ser intimidado en uno $u$ otro momento por racismo, por tener menor capacidad cognitiva, por alguna necesidad educativa especial, o por poseer una diferencia física que le aleja del restos de iguales.

Desde el punto de vista socio-emocional, el perfil de las víctimas tiende a caracterizarse por niveles altos de ansiedad, baja autoestima, inseguridad, fragilidad emocional, depresión, exclusión social, ausentismo escolar, trastornos psicosomáticos, o ideas suicidas.

Algunas de las manifestaciones más comunes en la salud física de las víctimas se traducen en dolores de cabeza, trastornos alimenticios, acné, ataques de pánico, sudoración, 
temblor, trastornos de sueño, enuresis, dolores estomacales, aceleración en las palpitaciones, soriasis, úlceras, entre otras.

Si el maltrato escolar se presenta en poblaciones de adolescentes y, sobre todo, si las víctimas son mujeres pueden desarrollarse trastornos alimenticios y sentimientos negativos con repercusiones en otros ambientes y contextos y, a nivel escolar, se manifiesta con el ausentismo, el bajo rendimiento académico así como el aislamiento. Como puede apreciarse en la Tabla y Gráfico 3, en los tres estudios se evidencia a una persona que ejerce un doble rol de víctima y victimario. Este rol se presenta como respuesta a una agresión previa, y responde a la intimidación defendiéndose, pero debe tenerse especial cuidado hacia ellas, ya que se considera la población más propensa a manifestar conductas agresivas, porque muestran más dificultad para regular sus emociones, baja autoestima, condiciones que motivan, en algunos casos, a reacciones imprevistas, porque no logran controlar el enojo y la ira.

De acuerdo con el estudio realizado, las víctimas que responden a sus victimarios, se muestran muy reactivas y contestan ante cualquier acto agresivo aunque no sea intencional, mostrando inseguridad y poco apego por el centro educativo.

Otro aspecto que se investigó en este estudio fue el de si los acosadores o acosadoras en el momento de agredir a sus iguales se encontraban solos o acompañados. Estos datos pueden apreciarse en la Tabla y Gráfico 5.

Tabla 5

Condición del estudiante al momento de acosar a sus pares durante el 2007, 2010 y 2012 , tomando en cuenta el rango de edad de la muestra

\begin{tabular}{|c|c|c|c|c|c|}
\hline \multicolumn{2}{|c|}{$\begin{array}{l}\text { Entre } 13 \text { y } 16 \text { años } \\
(2007)\end{array}$} & \multicolumn{2}{|c|}{$\begin{array}{c}\text { Entre } 9 \text { y } 14 \text { años } \\
(2010)\end{array}$} & \multicolumn{2}{|c|}{$\begin{array}{c}\text { Entre } 9 \text { y } 14 \text { años } \\
(2012)\end{array}$} \\
\hline Acompañado (a) & $61 \%$ & Acompañado (a) & $52 \%$ & Acompañado (a) & $19 \%$ \\
\hline Solo (a) & $39 \%$ & Solo (a) & $39 \%$ & Solo (a) & $13 \%$ \\
\hline
\end{tabular}




\section{Gráfico 4 \\ Condición del estudiante al momento de acosar a sus pares durante el 2007, 2010 y 2012, tomando en cuenta el rango de edad de la muestra}
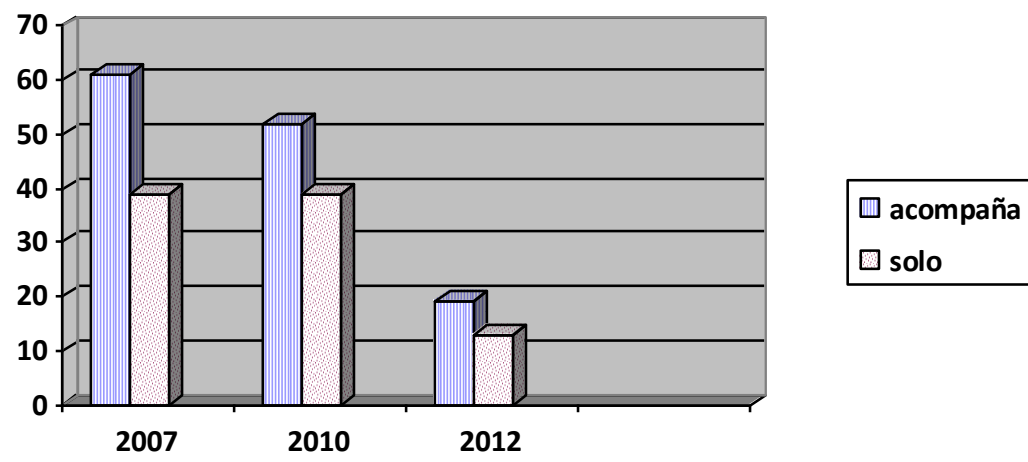

\section{Comentario}

Como puede apreciarse, existe una tendencia de la persona victimaria de intimidar a sus iguales, acompañándose de otros, como una forma de diluir la responsabilidad, para que, en caso de ser descubierto, el peso del castigo no recaiga sobre él (ella), sino que la culpa sea repartida. De esta manera, se diluye la responsabilidad del hecho, acción que probablemente disminuiría los efectos de la sanción aplicada. Esto podría interpretarse de forma que, mediante el acto de agresión, la persona que maltrata, además de buscar intimidar de manera intencionada, busca, a la vez, reafirmar un rol social dominante, conducta que se ve reforzada por los pares que lo presencian.

Este tipo de agresor completa su necesidad de llamar la atención y de mantener su estatus de popularidad entre el grupo, por medio de la manifestación de conductas violentas contra aquellos compañeros y compañeras que son percibidas como más débiles.

También, la presencia de los espectadores se convierte en un refuerzo positivo para que la conducta agresiva siga manteniéndose, ya que ante los ojos de los iguales la persona que maltrata queda como "el/la más fuerte", o como "quien tiene el control de la situación", lo que podría generar, en quienes observan la situación, sentimientos de respeto y temor, y en las víctimas el de humillación y baja autoestima.

En la intimidación entre compañeros y compañeras se perciben las características individuales, como reflejo del texto social de cada niño y niña, que se contrapone con el ambiente que se vive en la escuela. 
Los observadores juegan un rol fundamental, porque algunos incitan a la agresión y apoyan al victimario, reforzando los actos que ejerce, pero también están aquellos que solo observan y no hacen nada para defender a las víctimas.

Es importante anotar que los observadores no siempre son los niños o las niñas, también pueden ser docentes u otros miembros de la comunidad estudiantil donde se desarrollan los hechos. Al no intervenir, de igual manera están validando las acciones cometidas, volviendo a las víctimas más vulnerables, y postrándolas en una total indefensión, ya que se supone que quienes deben defenderles no lo hacen. Al existir negligencia, por parte del personal docente o de las instituciones en general, la escuela se convierte en responsable de los hechos y debe asumir las consecuencias que conllevan estos actos.

El nivel de conciencia social que ha de desarrollarse en los centros educativos, para no permitir que estos actos degradantes formen parte de la cotidianidad de los estudiantes en las relaciones de aula, es una labor apremiante, que requiere del esfuerzo conjunto que involucre a la familia, a la escuela y a la comunidad.

En muchos casos, cuando los niños y niñas denuncian los hechos, la intimidación no termina, pero puede disminuir como se aprecia en los porcentajes que se presentan en la Tabla 6.

Tabla 6

Porcentajes de respuestas obtenidos sobre: si una vez denunciados los hechos, continuaron las amenazas, por edades en los años 2011 y 2012

\begin{tabular}{|c|c|}
\hline $\begin{array}{c}\text { Entre } 9 \text { y } 14 \text { años } \\
(2011)\end{array}$ & $\begin{array}{c}\text { Entre } 9 \text { y } 14 \text { años } \\
(2012)\end{array}$ \\
\hline Sí $24 \%$ & Sí $12 \%$ \\
\hline No $56 \%$ & No $24 \%$ \\
\hline
\end{tabular}

\section{Comentario}

Si bien algunos estudiantes denuncian los hechos, no siempre los actos vandálicos hacia ellos disminuyen, porque aumenta su vulnerabilidad, al quedar expuestos ante las personas acosadoras, quienes al ser descubiertos dirigen los ataques hacia sus víctimas con mayor hostilidad. En los centros educativos se ha instalado "una ley del silencio", y es por ello que un porcentaje alto de la población estudiantil victimizada, no denuncia los hechos, por lo que familiares y docentes ignoran la situación que se está presentando. 
Otro factor que incide en este silencio es aquel en el que existe un porcentaje muy alto de victimarios que no reciben ningún castigo, hecho que es conocido por las víctimas, quienes prefieren callar antes de sufrir mayores agresiones, vandalismo o aislamiento.

\section{Conclusiones}

El acoso escolar en la escuela costarricense es un fenómeno que va en aumento, como se percibe a través de los diferentes estudios realizados, por lo que, no solo debe verse como un fenómeno generado entre el alumnado, sino como una situación integral que involucra a la familia, a la escuela y a una sociedad, problema que, a su vez, tiene graves consecuencias en el desarrollo socio emocional que, además, incide en la salud de la población involucrada.

Entre algunas de las causas que podrían estar aumentando el acoso entre iguales en la escuela costarricense podrían mencionarse el deterioro de la situación económicas de muchos hogares, lo que lleva a una desigualdad social en los habitantes, así como la crisis de valores existente entre la población estudiantil matriculada en el sistema educativo.

La diferencia de un 6\% en los años 2010 y 2012 de las personas que dicen victimizar a sus compañeros, es un indicador al que debe prestársele especial atención; sin embargo, el porcentaje anotado por quienes manifiestan ser víctimas de sus compañeros es más alarmante aun, ya que de acuerdo con los datos obtenidos creció de un $29 \%$ en el 2010 a un $41 \% 2012$.

Estos datos llevan a la siguiente interrogante: ¿es la población estudiantil más agresiva conforme transcurre el tiempo?, o, ¿ha pasado la violencia a ser parte de la cultura de la población estudiantil costarricense?

De ser así, pareciera que el maltrato entre iguales se está convirtiendo en una parte importante en la convivencia entre los niños y las niñas, quienes se unen entre ellos en los centros educativos, buscando identidad, o para pertenecer a grupos bajo reglas que respetan, siguiendo normas que rigen sus comportamientos, convirtiéndose éstas en creencias sobre las que actúan.

Los datos encontrados muestran la existencia de víctimas y victimarios, además de otros actores como los observadores, identificados y aceptados como parte de la dinámica escolar. Al establecerse esta mecánica en los salones de clase, se confirma que los pares juegan una función instrumental en la intimidación de otros, haciéndoles creer a quienes 
agreden, que su conducta es positiva, y la mantienen como una forma de fortalecer el estatus.

En general, la población costarricense muestra rechazo y se conmueve ante las publicaciones en los diarios nacionales de hechos acaecidos sobre el acoso en el aula, pero, paralelo a ello, también hay evidencia sobre la falta de límites por parte de figuras de autoridad para guiar los comportamientos de sus estudiantes, tanto en el aula como en el hogar, convirtiéndose este aspecto en un factor desencadenante del fenómeno del bullying en el aula.

La posibilidad de que los niños y las niñas reproduzcan patrones aprendidos en el hogar, como los gritos, comentarios nocivos, las burlas y hasta los golpes, o los asuman como normas de comportamiento, es muy alta, por lo que el control de las conductas amenazantes debe trabajarse primero en el seno familiar y después retomarse en la escuela, por lo que ha de realizarse una labor conjunta escuela-hogar, donde se concientice a las madre y a los padres respecto a que los hijos y las hijas no viven en forma aislada, sino que son parte de un sistema social donde deben desarrollarse.

Es de vital importancia fomentar el asesoramiento a padres y madres, sobre todo, en aquellas personas que utilizan el castigo físico, los gritos, los insultos o la indiferencia al educar, creyendo que son los parámetros idóneos para corregir a los niños y a las niñas, por lo que debe enfatizarse al respecto para que se puedan cambiar los patrones de crianza.

Por los datos obtenidos, puede apreciarse que existe en la escuela costarricense un problema de inseguridad que afecta el aprendizaje de la población estudiantil, ya que al no poder desarrollar plenamente sus habilidades, debido al clima de temor y desesperanza que viven los y las estudiantes, que les lleva a retraerse, aislarse o a sucumbir ante los victimarios, cayendo en estados de depresión injustificables desde edades tempranas, donde la única misión de la niñez es la de gozar de libertad y seguridad, con un solo objetivo, ser feliz.

\section{Recomendaciones}

Brindar asesoramiento e instrumentar con programas al personal docente en servicio para que puedan ser implementados en el aula, de tal forma que les permitan abordar el bullying en los diferentes niveles del sistema educativo, iniciando en Preescolar, Primaria y terminando en Secundaria. 
Si bien es cierto el fenómeno del acoso escolar tiende a disminuir en los niveles superiores, debe darse seguimiento tanto a las víctimas como a los victimarios, así como a sus familias, para un mejor control de la situación, desde los niveles iniciales.

Debe hacerse conciencia entre los padres y madres, así como en figuras de autoridad acerca de que los niños y las niñas, como personas, no solo se desarrollan en el seno familiar, sino que forman parte de una comunidad que, en algunos casos, les protege, pero que también les excluye dependiendo de los comportamientos, y habilidades que adquieran para convivir en ella.

Incluir en los talleres formativos evidencias que le permitan reconocer al docente la problemática del acoso escolar, saber que es un fenómeno real que está afectando a un porcentaje muy alto de los niños y las niñas costarricenses.

La actitud y buena voluntad por parte del y la docente para resolver las situaciones conflictivas en el aula no son suficientes para resolver las conductas agresivas en el aula, porque el abordaje de esta temática debe desarrollarse a través de programas estructurados que le brinden capacitación, acerca de la teoría existente, así como de diferentes formas para abordar el fenómeno.

\section{Referencias}

Aluede, Oyaziwo; Adeleke, Fajaju; Omoike, Don; y Afen-Akpaida, Justina. (2008). A Review of the Extent, Nature, Characteristics and effects of Bullying Behaviour in Schools. Journal of Instructional Psychology, 35(2), 151-158.

Brockenbrough, Karen; Cornell, Dewey G.; y Loper, Ann B. (2002). Aggressive attitudes among victims of violence at school. Education and Treatment of Children, 25, 273-287.

Cabezas, Hannia. (2007). Detección de conductas agresivas "Bullying" en escolares de sexto a octavo año, en una muestra costarricense. Educación: Revista de la Universidad de Costa Rica, 31(1), 23-133.

Cabezas, Hannia y Monge, Irene. (2007). Maltrato entre iguales en la escuela costarricense. Educación: Revista de la Universidad de Costa Rica, 31(1), 135- 144.

Cabezas, Hannia. (2010). ¿Qué ocurre en el aula costarricense? Los niños y las niñas que maltratan a sus compañeros. Actualidades Investigativas en Educación, 10(3), 1-21.

Cabezas, Hannia. (2011). Los niños rompen el silencio. Estudio exploratorio de conductas agresivas en la escuela costarricense. Educación: Revista Universidad de Costa Rica, 35(1), 1-21. 
Cid, Patricia; Díaz, Alejandro; Pérez, María Victoria; Torruella, Matilde y Valderrama, Milady. (2008). Agresión y violencia en la escuela como factor de riesgo del aprendizaje escolar. Ciencia y enfermería XIV, 2, 21-30.

Craig, Wendy; Harel-Fisch, Yossi; Fogel-Grinvald, Haya; Dostaler, Suzanne; Hetland, Jorn; Simons-Morton, Bruce; et al. (2009). A cross-national profile of bullying and victimization among adolescents in 40 countries. International Journal of Public Health, 54, 216-224.

Currie, Candace E.; Gabhinn, Saoirse; y Godeau, Emmanuelle. (2008). Inequalities in young people's health. Health behavior in school-aged children: international report from the 2005, 2006 survey. Health Policy for Children and Adolescents, 5. University of Edinburg: Child and Adolescent Health Research Unit, Cahru. Recuperado de http://www.education.ed.ac.uk/cahru

Díaz-Atienza, Francisco; Prado Cuesta, Mercedes y Ruiz-Veguilla, Miguel. (2004). Relación entre las conductas de intimidación, depresión e ideación suicida en adolescentes: resultados preliminares. Revista de Psiquiatría y Psicología del Niño y del Adolescente. 4(1), 10-19.

Espelage, Dorothy. (2003). La intimidación en el comienzo de la adolescencia: La función del grupo social. Eric Digest, 3(2). Recuperado de http://www.ericdigests.org/20034/grupo.html

Farrow, Claire V. y Fox, Claire L. (2011). Gender differences in the relationships between bullying at school and unhealthy eating and shape-related attitudes and behaviors. British Journal of Educational Psychology, 81, 409-420.

García Continente, Xavier; Pérez Giménez, Anna y Nebot Adell, Manuel. (2010). Factores relacionados con el acoso escolar (bullying) en los adolescentes de Barcelona. Gac Sanit, 24(2), 103-108.

Gwen, Dewar. (2008). When bullies get bullied by others: Understanding bully-victims. Recuperado de http://www.parentingscience.com/attachment-parenting.html

Kumpulainen, Kirsti y Räsänen, Eila. (2000). Children Involved in Bullying at Elementary School Age: Their Psychiatric Symptoms and Deviance in Adolescence. An Epidemiological Sample. Child Abuser Neglect, 24, 1567-1577.

Loredo, Arturo; Perea, Arturo y López, Gloria Elena. (2008). "Bullying": acoso escolar. La violencia entre iguales. Problemática real en adolescentes. Acta Pediátrica de México, $(29,4), 210-214$.

Menesini, Ersilia y Nocentine, Annalaura. (2009). El acoso en la escuela. En Francesco Mazzone y Querciolo Mazzonis (compiladores), Educación en Contextos de violencia y violencia en contextos educativos. Comitato Internazionale per lo Sviluppo dei Popoli. Roma, Italia. 
Monclús, Antonio. (2005). La violencia escolar: Perspectivas desde Naciones Unidas. Revista Iberoamericana de Educación, 38, 13-32.

Nansel, Tonja R.; Overpec, Mary; Pilla, Ramani; Ruan, W.June; Simons- Morton, Bruce; y Scheidt, Peter. (2001). Bullyings behaviors among US youth: Prevalence and association with psychological adjustment. Journal of the American Medical Association, 285, 2094-2100. Recuperado de http://pdba.georgetown.edu/Security/citizensecurity/eeuu/documents/bullying.pdf

Navarro Olivas, Raúl. (2009). Factores psicosociales de la agresión escolar: La variable género como factor diferencial. (Tesis doctoral). Universidad Castilla de la Mancha, España. Recuperado de https://ruidera.uclm.es/xmlui/bitstream/handle/10578/1005/273\%20Factores\%20psicos ociales\%20de\%20la\%20agresi\%C3\%B3n.pdf?sequence=1

Ng, Josephine W.Y. y Tsang, Sandra K.M. (2008). School Bullying and the Mental Health of Junior Secondary School Students in Hong Kong. Journal of School Violence, 7(2), 320.

O’Brennan, Lindsey; Bradshaw, Catherinne P. y Sawyer, Anne L. (2009). Examining developmental differences in the social-emotional problems among frequent bullies, victims, and bully/victims. Psychology in the schools, 46(2), 100-115.

Olweus, Dan. (1993). Bullying at School. What we Know and What we can Do. Cambridge, M.A.: Blackwell.

Pincever, Karina. (2008). Maltrato infantil. El abordaje innovador del Programa IELADEINU con el apoyo de UNICEF. Argentina: Editorial y distribuidora Lumen, SRL.

Salmivalli, Christina; Lagerspetz, Kirsti; Bjorkqvist, Kaj; Osterrman, K. y Kaukiainen, Ari. (1996). Bullying as a group process: Participant roles and their relation status within the group. Aggressive Behavior, 22, 1-15.

Smokowski, Paul R. y Kopasz, Kelly Holland. (2005). Bullying in school: An overview of types, effects, family characteristics, and intervention strategies. Children and schools, 27(2), 101-110.

Sourander, Andre; Jensen, Peter; Rönning, John A; Niemelä, Solja; Helenius, Hans; Sillanmäki, Lauri; Kumpulainen, Kirsti; Piha, Jorma; Tamminen, Tuula; Moilanen, Irma; y Almqvist, Frederik. (2007b). What is the early adulthood outcome of boys who bully or are bullied in childhood? The Finnish "From a Boy to a Man" study. Pediatrics, 120(2), 397-404.

Sullivan, Keith; Cleary, Mark y Sullivan, Ginny. (2005). Bullying Secondary Schools: What it looks like and how to manage it. SAGE Publications Ltd. 